\title{
E-Learning Barriers and Solutions to Knowledge Management and Transfer
}

\author{
*Oye, N. D, Salleh, M, A.Iahad, N. \\ Universiti Teknologi Malaysia \\ *oyenath@yahoo.co.uk
}

\begin{abstract}
This paper present a systematic overview of barriers and solutions of e learning in knowledge management (KM) and knowledge transfer (KT) with more focus on organizations. The review paper also discusses KT in organizational settings and KT in the field of e learning. Here, an e-learning initiative shows adaptive solutions to overcome knowledge transfer barriers. The paper concludes that the flow of knowledge depends on people and the social environment they operate in. Therefore E-Learning environments support knowledge transfer not only by helping learners to make sense of content, but also by enabling communication, both among learners and between learners and tutors.
\end{abstract}

Keywords: Knowledge Management (KM), Knowledge Transfer (KT), E-learning, Learning Object (LO), Learning Resource (LR).

\section{Introduction}

Knowledge and its efficient management constitute the key to success and survival for organizations in the highly dynamic and competitive world of today. Efficient acquisition, storage, transfer, retrieval, application, and visualization of knowledge often distinguish successful organizations from the unsuccessful ones, and are essential for management of knowledge. Knowledge management (KM) is not about technology, it is about people. People as individuals, people as teams, people as communities, and people as organizations. If technology is handled properly, it can make an important contribution. Technology can connect people with each other, it can connect people to information they find useful and it can protect them from irrelevant content. There are numerous discourses associated with KM but it is only in recent times that e learning has been identified as a strategic resource that can be utilized in an increasing diversity of venues (home, workplace, cultural and entertainment venues, as well as traditional institutions of learning, education, and training).

Learning and knowledge have a symbiotic relationship, they depend upon each other. From a slightly more complex perspective the creation, acquisition, transfer, and exchange of knowledge are all activities that are helping define the character of information - and knowledge-based economies - in which the primary assets of data, information, and knowledge all manifest digitally. The technological tools facilitating much of these interactions are information and communication technologies (ICT). Moreover, it is through engaging with ICT that learning defines itself as e learning. During the last century, we have moved from the Industrial Age through the Information Age and now, to the Knowledge Age. The ability to obtain, assimilate, and apply the right knowledge effectively will become a key skill in the next century. Learning is the key to achieving our full potential. In fact, our survival in the 21st century as individuals, organizations, and nations will depend upon our capacity to learn and the application of what we have learned to our daily lives. In this paper, present Section 2 discusses the definition of e learning and the history of e learning. Section 3 discusses knowledge management as a concept and a scientific discipline. Section 4 discusses knowledge transfer; here KT is promoted as a social and interpersonal activity. Section 5 focus on knowledge transfer barriers and section 6 examines solutions to barriers of knowledge transfer. Section 7 concludes that the flow of knowledge depends on people and the social environment they operate in.

Definition of E-learning: The history of e learning occurs in a more recent timeframe. The development of telecommunications technology and personal computers provides what is considered the general timeline of e learning. The timeframe of greatest development in this area is thus within the last forty years .An early example of what we might define as e learning occurred in 1909, when Robert E. Peary, arctic explorer, radiotelegraphed: "I found the Pole". Combining the characteristics of communication technology with an explicit 
educational objective, the knowledge that he has found the North Pole. Peary inadvertently produced an elearning occurrence for his listeners. However, as electronic equipment has been developed, specifically the microprocessor and personal computer, it too has been implicated in learning, culminating in current e learning. Therefore, the history of e learning runs parallel with the development of electronic equipment and the use of information and communication technologies.

Globalization is focused on e learning because e-learning technology has the potential to bring improved learning opportunities to a larger audience than has ever previously been possible. (Sharma, Ekundayo, \& Ng, 2009) suggested that a nation's route to becoming a successful knowledge economy is its ability to also become a learning society. Students' learning in tertiary institutions all over the world has undergone tremendous transformation, especially since the advent of information and communication technology (ICT). (Czerniewicz \& Brown, 2009) argued that globally, higher education has been required to become responsive to many more social interests than was previously the case and to engage with the imperatives being voiced by many different groups ranging from unions and associations to industry, business and regional authorities. (Erah, 2006) stated that the term e learning refers to computer-enhanced training as opposed to the computer-based training of the 1980s. It is usually delivered in a personal computer and includes learning delivered by other communications technologies. According to him, e learning is an approach to facilitate and enhance learning through both computer and communication technologies. The devices often used for this purpose include personal computers, CD ROMs, television, personal digital assistants (PDAs - handheld devices that were originally designed as personal organizers, but became much more versatile over the years), MP3 players and mobile phones. Communication technology enables the use of internet, e-mail, discussion forums, collaborative software, classroom management software, team learning systems, intranet, extranet, Local Area Network (LAN), Wide Area Network (WAN), audio and videotape, satellite and interactive television lectures, satellite-delivered learning, virtual educational networks, satellite downlinks, computerized diagnostic assessment, competency certification and electronic portfolios(Kaplan-Leierson, 2006; Oye, Salleh, \& Iahad, 2010).

\section{Knowledge Management (KM)}

KM comprises a range of strategies and practices used in an organization to identify, create, represent, distribute, and enable adoption of insights and experiences. Such insights and experiences comprise knowledge, either embodied in individuals or embedded in organizational processes or practice. Many large companies and non-profit organizations have resources dedicated to internal KM efforts, often as a part of their 'business strategy', 'information technology', or 'human resource management' departments (Addicott, McGiven, \& Ferlie, 2006). Several consulting companies also exist that provide strategy and advice regarding KM to these organizations. Knowledge management efforts typically focus on organizational objectives such as improved performance, competitive advantage, innovation, the sharing of lessons learned, integration and continuous improvement of the organization. KM efforts overlap with organizational learning, and may be distinguished from that by a greater focus on the management of knowledge as a strategic asset and a focus on encouraging the sharing of knowledge. KM efforts can help individuals and groups to share valuable organizational insights, to reduce redundant work, to avoid reinventing the wheel per se, to reduce training time for new employees, to retain intellectual capital as employees turnover in an organization, and to adapt to changing environments and markets(Thompson \& Walsham, 2004).

Early KM technologies included online corporate yellow pages as expertise locators and document management systems. Combined with the early development of collaborative technologies (in particular Lotus Notes), KM technologies expanded in the mid-1990s. Subsequent KM efforts leveraged semantic technologies for search and retrieval and the development of e-learning tools for communities of practice. Knowledge in modern economies is increasingly playing a key role in achieving organizational success. Knowledge management as a concept and a scientific discipline emerged to acknowledge this fact. Three main reasons can be identified for these developments are: 
- Need: Today's information technology enabled organizations to process and make use of more information in ever-decreasing time cycles.

- Recognition of need: Organizations increasingly recognize the need for and the importance of conscious management of knowledge.

- Availability of KM-Instruments.

Practicing knowledge management in organizations can be achieved through the development and implementation of knowledge infrastructures as shown in Figure 1. Here knowledge infrastructures are defined as the set of all successfully implemented interventions, measures, institutions and facilities that represent a supportive knowledge environment for knowledge workers who execute knowledge intensive tasks.

\section{Figure 1: Strategic Perspective on Knowledge Infrastructures}

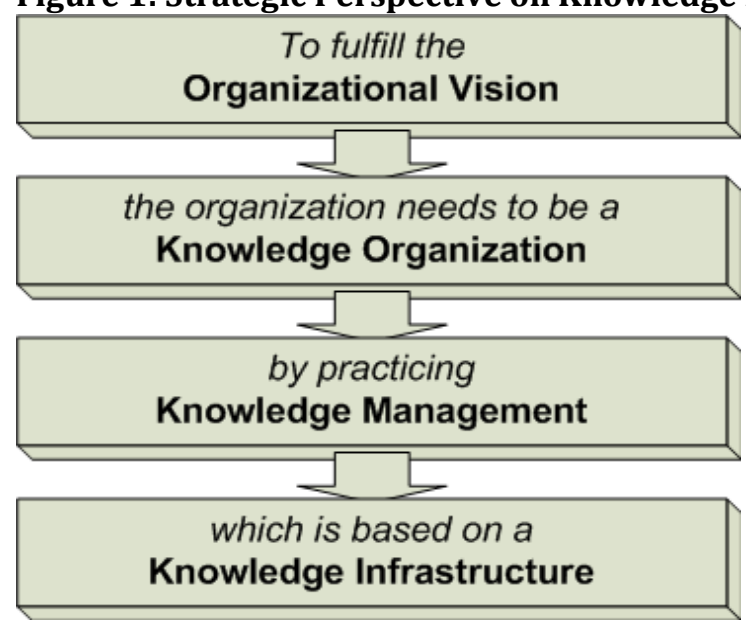

KM deals with the conscious management of organizational knowledge through knowledge management interventions. Organizational knowledge is defined as information that is relevant for executing certain (business) actions. KM interventions are the set of all potential organizational initiatives that aim to improve an organization's knowledge infrastructure. They can become, if successfully deployed, an integral part of knowledge infrastructures

\section{Knowledge Transfer (KT)}

The concept of KT is one aspect of the larger KM discipline. The movement of knowledge - including knowledge based on expertise or judgment, from one person to another. This definition emphasizes the exchange of tacit knowledge through personal connections and that knowledge is embedded in work practices and bound by individually based experiences, judgments, and skills. As a result, it is impossible to fully capture it in a report or PowerPoint slide. While it is important to be able to discover and access explicit information, it is the actual transfer of tacit knowledge that is vital to increased learning and knowledge within an organization as shown in Figure 2. KT is enabled through social interactions among people within formal and informal networks. It is beneficial to facilitate the establishment of personal connections to increase knowledge flow within an organization. This is based on the understanding that it is not only what you know but also whom you know. 
Figure 2: Transfer of tacit knowledge

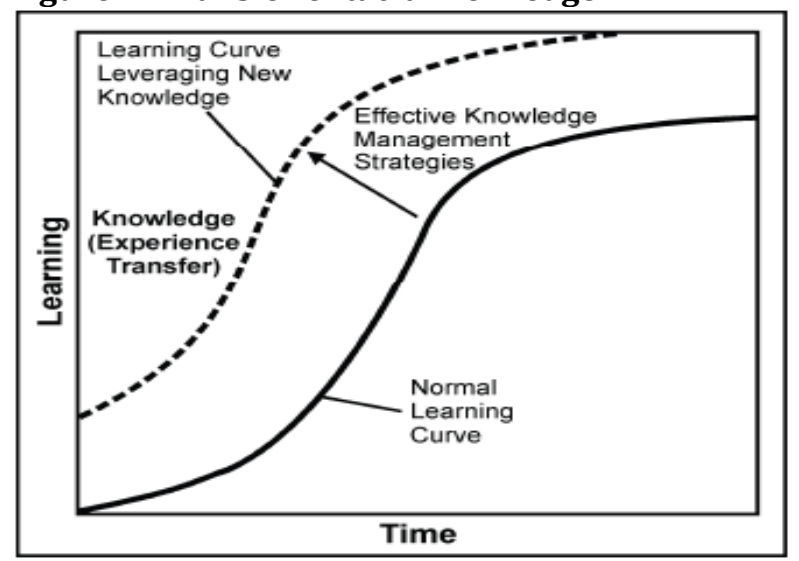

KT is studied from multiple perspectives including the fields of e learning and knowledge management. More recently, many papers specifically target the integration of findings from the two fields (Maier \& Thalmann, 2007) in order to foster knowledge transfer between persons, organizational units or entire organizations or institutions respectively. These learning processes can be supported by information and communication technologies (ICT), typically called e learning. E-learning resources, that are resources documenting knowledge refined with the help of didactical methods, can be prime instruments for knowledge transfer. Documented knowledge exists in differed forms, atomic as smallest unit of explicit documented knowledge, called knowledge chunks or as composed grouping of formatted information objects which cannot be separated without substantial loss of meaning, called documents (Maier, 2007).

Knowledge Transfer Principles: Knowledge is always tacit to begin with, that is, it exists only in workers' mind and cannot be articulated easily. The struggle is to articulate this knowledge and transfer it into a physical medium so that it can be distributed, reuse and perhaps serve as the foundation of new knowledge.

Exploit Tacit Knowledge: Information captured in digital form, on paper, and in pictures generally tells what and why, but not how. Tacit knowledge explains the "how" and resides in individuals. It includes experience and expertise gained from operations and training, learned nuances and subtleties, and work- rounds. Mental agility, effective responses to crises, and the ability to adapt to change are also forms of tacit knowledge. This knowledge form is the domain of individuals, not technology.

Promote KT as a social and interpersonal activity: The process of KT can be enhanced through participation in interpersonal activities that are designed to expand shared experiences. Relationship building activities further the goals of creating a knowledge-sharing environment. Improved organizational effectiveness, operational processes, and decision-making are results of a knowledge sharing culture. This requires a culture in which people seek out others who have the knowledge and actively build their personal social network to improve knowledge sharing.

Connect people with expertise: KT occurs when individuals are able to connect with others who have the desired expertise and experiences. Being able to leverage one's personal network through awareness and information technology can aid individuals who seek out, and desire to connect with the appropriate experts. People learn from both their personal experiences and through the experiences shared by others. Organizational learning strategies should encourage interactions and collaboration that promote knowledge transfer among individuals in organization. Fostering learning produces organizations and personnel who are able to learn faster than enemies are and adversaries do.

Promote trust and mutual understanding: Successful knowledge transfer depends on willingness to share knowledge so that others can benefit. This requires trust and understanding on both personal and professional level. Organizational initiatives should seek to increase trust and understanding among people in the organization to build stronger relationships. In the scope of e-learning, documented knowledge that can 
be used for learning purposes is typically called a learning resource (LR) or learning object (LO). A large number of initiatives have been deployed that specifically target knowledge transfer from developed to developing countries. Case studies have encountered significant barriers that have to be overcome, e.g., due to heterogeneous target groups for learning. Main differences are learning styles, motivation, age, previous knowledge, religion, cultural background and sex. A number of techniques have been developed for creating adaptable and adaptive e-learning systems (Brusilovski, 2001) which could provide partial solutions to this challenge.

Knowledge Transfer Barriers: KT problems with the delivery of LRs from developed to developing countries can be the result of barriers between designers of LRs and receivers. Such barriers can be related to, e.g., the infrastructural context, the cultural context, and the transferred knowledge (Maier, 2007). ICTs used in education are generally seen as a factor for national development and, therefore, a fundamental dimension. Regarding the case studies, barriers for knowledge transfer with LRs to developing countries can be distinguished in infrastructure, technology access, Internet access, maintenance of technology, and usability barrier (Giannini-Gachago \& Seleka, 2005; Hodgins-Williams \& Mostert, 2005; Hodgins-Williams, Sieborger, \& Terzoli, 2007; Lee, Thurab-Nkhosi, \& Giannini-Gachago, 2005; Lumande, Ojedokun, \& Fidzani, 2006; Muwanga-Zake, 2007; Naqvi, 2006; Wells \& Wells, 2007). The infrastructure barrier comprises aged infrastructure such as old landlines and lack of electricity. For example, privatization of the power sector often led to a lack of power in 6 of 7 observed schools in Uganda (Wells \& Wells, 2007). Even if infrastructure is existent, services can be problematic, such as in South Africa where bills were not paid by schools and as a consequence they were disconnected from the Internet (Muwanga-Zake, 2007). The technology access barrier results from limited, absent or insufficient access to hard- and software. The access to technology in developing countries is low, in schools as well as at home (Giannini-Gachago \& Seleka, 2005). Computer laboratories are not always open to students. The maintenance barrier covers installation and maintenance of equipment, such as hard- and software. Maintenance is difficult due to the fact that the specialists who implemented the system had returned to their home countries and were not available anymore (Wells \& Wells, 2007).

Culture can be defined "as the collective programming of the mind that distinguishes the members of one group or category of people from another. Therefore, people treat LRs and internalize content differently based on their backgrounds. The language barrier is an important aspect of transforming LRs to developing countries. "Language is the most clearly recognizable part of culture. Besides LRs, software, and most Web contents are in English and that is a low level of language skills in developing countries, which can lead to misunderstandings. For example, the term 'mouse' which in ordinary English denotes an animal had to be translated to reflect a computer device. The social context barrier involves typical local opinions and traditions of a society Knowledge or group. Ubuntu, an African culture, has a specific value system and social conducts (Muwanga-Zake, 2007) which have to be considered. For many Africans, the Internet is an entertainment and communication tool and e learning is for those who failed in traditional schools or those who have sufficient money to pay for international education. The didactical barrier can be seen because of procedures defined in a curriculum conflicting with procedures that students and teachers were used to before the e-learning initiative and a lack of openness to new learning approaches. For example, students and teachers had no more or rare face-to-face contact which was new to Thai students.

The computer literacy barrier covers the level of computer and information skills in developing countries, which is generally low. Special training is necessary to acquire these skills. Students and teachers had little or even no experiences using a computer. Even basic computer skills, such as using a mouse were absent among most teachers (Muwanga-Zake, 2007). The competence barrier results from little or no previous knowledge for handling and understanding LRs. Most teachers never had experiences in using computers to teach. Sometimes, instructors and assistants were not understood well by students and searching for materials on their own was new for some Thai students.

\section{Solutions to Barriers of knowledge Transfer}

E-learning courses mostly are containers comprising resources, like text fragments, pictures, animations or videos, called media objects (MOs). Compositions of MOs enriched by the application of didactical methods or 
techniques are called learning objects (LOs). Wiley defines a LO openly as any digital resource that can be reused to support learning. In addition to the link to learning, this definition focuses on reuse as a key characteristic of LOs. In the scope of e learning, a broad variety of metadata standards and specifications exist. Thereby, three main application domains can be distinguished: (i) metadata for LOs, (ii) metadata for learner descriptions and (iii) metadata for content aggregation. Metadata for LOs primarily describe content and usage of LOs. The dominant standard in this field is IEEE Learning Object Metadata (http://ltsc.ieee.org/wg12/files/LOM_1484_12_1_v1_Final_Draft.pdf)(Duval, 2004). Metadata for learner descriptions describe the learner itself and are primarily used for adaptation and evaluation.

Alternatives for adaptation cannot provide sufficient solutions for every technology transfer barrier. In many cases, it is primarily investments in technical equipment that is needed(Giannini-Gachago \& Seleka, 2005). In order to successfully apply e learning in general and consequently alternatives for adaptation, a minimal level of technical infrastructure must be available. In the case of infrastructure and technology access barrier, we can consider that the technical infrastructure is aged and cannot process LRs. MOs have to be modified so that the existing infrastructure can cope with it. For instance, resolutions of graphics and videos must be reduced or content has to be reformatted. Furthermore, MOs inside an LO that cannot be processed must be removed and if possible replaced by processible MOs.

In the case of an Internet access barrier, provisions similar to the ones mentioned in the case of infrastructure barrier can be used. If no Internet connection is available, LRs should be provided on data carriers, like CD or DVD, as CBT or static WBT consisting of static HTML code. Therefore, SCORM containers including LRs and sequencing descriptions, e.g., simple sequencing information can be used. In case of very low bandwidth, hybrid methods can also be applied. Transfer problems resulting from insufficient interfaces, called usability barrier, can be overcome by redesigning the interfaces. Normally, the interface is provided by the Learning Management System rather than by the LR itself. The interaction barrier is not primarily focused on the content itself. Instead, the way of how learners interact and discover the LR is critical. For problems not directly related to the LR, like interaction problems with online communication, reported in a South African case study, adaptation does not offer appropriate support. However, organizational provisions, like role concepts or incentives to participate can help to overcome this barrier. Barriers concerning previous knowledge can be coped with by providing the learners with additional information enabling them to absorb LRs. Concerning the computer literacy barrier,(Giannini-Gachago \& Seleka, 2005; Hodgins-Williams \& Mostert, 2005; Lee et al., 2005) recommend training learners in computer usage, such as frequenting orientation sessions or passing a prerequisite level of computer literacy.

\section{Conclusion}

E-learning barriers are heterogeneous encompassing personal, organizational, content, situation, instructional, and technological barriers. Knowledge management application is aimed to furnished organizations with tools to manage their business knowledge, while the focus of e learning has always been on managing the delivery of academic knowledge. This paper enumerated barriers to knowledge management and transfer as infrastructure context, cultural context, social context, didactical barrier, language barrier, computer literacy barrier and computer competence barrier. Certainly, there is no precise solution for every barrier in the technology dimension, where minimal technological infrastructure has to be there in order to develop a functioning e-learning solution on top.

The flow of knowledge depends on people and the social environment they operate in. As leaders and managers, it is important to understand the potential for improved performance by promoting a transfer culture within the organization. Communication technologies such as email, electronic discussion forums, instant messaging, chat rooms, and video conferencing primarily support knowledge transfer. E-Learning environments support knowledge transfer not only by helping learners to make sense of content, but also by enabling communication, both among learners and between learners and tutors. Knowledge transfer problems with the delivery of LRs from developed to developing countries can be the result of barriers between designers of LRs and receivers. 
Acknowledgement: The authors would like to thank all authors that their papers are quoted. In addition, the authors gratefully acknowledge UTM, Research Universiti Malaysia for their support and encouragement.

\section{References}

Addicott, R., McGiven, G. \& Ferlie, E. (2006). Networks, Organizational Learning and Knowledge Management: NHS Cancer Networks. Public Money \& Management, 26(2), 87-94.

Brusilovski, P. (2001). Adaptive hypermedia. User modeling and user adapted interaction. 11(1/2), 87-110.

Czerniewicz, L. \& Brown, C. (2009). A virtual wheel of fortune? Enablers and constraints of ICTs in Higher Education in South Africa. In M. W. K. a. W. T. E. S (Ed.), Bridging the knowledge Divide (pp57-76). Charlote: NC: Information Age.

Duval, E. (2004). Learning technology standadization: making sense of it all. Computer Science and Information Systems, 1(1), 33-43.

Erah, P. O. (2006). Intrduction to e-learning protocols. Paper presented at the ETF capacity-building workshop for lecturers of Universities in Nigeria at the University of Uyo.

Giannini-Gachago, D. \& Seleka, G. (2005). Experiences with international online discussions: Participation patterns of Botswana and American students in an adult education and development course at the University of Botswana. International journal of education and development using information and communication technology, 1(2), 163-184.

Hodgins-Williams, C. \& Mostert, M. (2005). Online debating to encourage student participation in online environments: a qualitative case study at a South African university. International Journal of Education and Development using ICT. 1(2), 94-104.

Hodgins-Williams, C., Sieborger, I. \& Terzoli, A. (2007). Enabling and constraining ICT practice in secondary schools: case study in South africa. International Journal of Knowledge and learning, $3((2 / 3)), 171$ 190.

Kaplan-Leierson, E. (2006). E-learning glossory [Electronic Version]. Retrieved 2006,

Lee, M., Thurab-Nkhosi, D. \& Giannini-Gachago, D. (2005). Using information collaboration to develop quality assurance processes for e learning in developing countries: the case of the university of Botswana and the university of West Indies Distance Education Centre. International journal of Education and Development using Information and Communication Technology, 2(1), 66-78.

Lumande, E., Ojedokun, A., \& Fidzani, B. (2006). Information literacy skills course delivery through WebCT: the university of Botswana Library experience. International Journal of Education and Development using ICT. 2(1), 66-78.

Maier, R. (2007). Knowlegde management systems. Information and communication technologies for knowlegde management. 3rd edn. Berlin: Springer.

Maier, R. \& Thalmann, S. (2007). Describing learning objects for situation oriented knowledge manament applications. Gronau, N. 4th (ed) (pp343-351) Paper presented at the Professional Knowledge management experiences and Visions, GITO, Berlin.

Muwanga-Zake, J. W. F. (2007). Introducing educational computer programmes through evaluation: a case in South African disadvantages schools. International Journal of Education and Development using ICT. $3(3), 30-48$.

Naqvi, S. (2006). Impact of WebCT on learning: an Oman experience. International Journal of Education and Development using ICT, 2(4), 18-27.

Oye, N. D., Salleh, M. \& Iahad, N. A. (2010). Holistic E-learning in Nigerian Higher Education Institutions. Journal of Computing, 2(11), 20-26.

Sharma, R., Ekundayo, M. S. \& Ng, E. (2009). Beyond the digital divide: policy anaylysis for knowledge societies. Journal of Knowledge management, 13(5), 373-386.

Thompson, M. P. A. \& Walsham, G. (2004). Placing Knowledge Management in Context. Journal of management studies, 41(5), 725-747.

Wells, R. \& Wells, S. (2007). Challenges and opportunities in ICT educational development: a Uganda case study. International Journal of Education and Development using IC. 3(2), 100-108. 